SMAD, Rev Eletrônica Saúde Mental Álcool Drog.

2021 abr.-jun.; $17(2): 82-91$

DOI: 10.11606/issn. 1806-6976.smad.2021.173019

www.revistas.usp.br/smad/

Artigo Original

\title{
Fadiga e uso de álcool por graduandos do curso de Enfermagem
}

Nathálie Dias de Oliveira Silva ${ }^{1}$

(D) https://orcid.org/0000-0001-7090-576X

Gabriel Fernandes Machado ${ }^{2}$

(D) https://orcid.org/0000-0003-3563-3492

Marcelle Aparecida de Barros Junqueira ${ }^{1}$

(D) https://orcid.org/0000-0002-2920-1194
${ }^{1}$ Universidade Federal de Uberlândia, Faculdade de Medicina, Uberlândia, MG, Brasil.

2 Universidade Federal de Uberlândia, Faculdade de Engenharia Elétrica, Uberlândia, MG, Brasil.
Objetivo: analisar o uso de álcool no padrão binge drinking e os níveis de fadiga em graduandos do curso em Enfermagem na Universidade Federal de Uberlândia. Método: a pesquisa é de abordagem quantitativa, utilizando estatísticas descritivas e bivariadas, com o valor de $p>0,05$, e realizada conforme a Resolução no 466/12 do CNS. Assim, foram aplicados questionários em uma amostra com 202 universitários da graduação em Enfermagem da Universidade Federal de Uberlândia. Resultados: o estudo mostrou a maior predominância do álcool no sexo feminino, com idade média de 22,6 anos de idade. Conclusão: a análise e a discussão dos dados possibilitaram afirmar que o uso abusivo de álcool está presente, principalmente na forma de binge drinking, e encontra-se associado à presença da fadiga e ao desempenho acadêmico nos universitários da graduação em Enfermagem, em diferentes períodos letivos.

Descritores: Estudantes de Enfermagem; Bebedeira; Fadiga; Consumo de Bebidas Alcoólicas; Saúde Pública; Adulto Jovem.

\section{Como citar este artigo}

Silva NDO, Machado GF, Junqueira MAB. Fatigue and alcohol use by undergraduate nursing students. SMAD, Rev Eletrônica Saúde Mental Álcool Drog. 2021 abr.-jun.;17(2):82-91. doi: https://dx.doi.org/10.11606/issn.1806-6976.smad.2021.173019 


\section{Fatigue and alcohol use by undergraduate nursing students}

Objective: to analyze the use of alcohol in the binge drinking pattern and the levels of fatigue in graduates of the Nursing course at the Federal University of Uberlândia. Method: the research is of quantitative approach, using descriptive and bivariate statistics, with the value of $p>0.05$, and carried out according to Resolution 466/12 of the NHC. Thus, questionnaires were applied to a sample of 202 university students from the Nursing undergraduate program of the Federal University of Uberlândia. Results: the study showed the highest predominance of alcohol in females, with a mean age of 22.6 years. Conclusion: the analysis and discussion of the data made it possible to affirm that the abusive use of alcohol is present, mainly in the form of binge drinking, and is associated with the presence of fatigue and academic performance in undergraduate students of Nursing, in different school periods.

Descriptors: Nursing Students; Binge Drinking; Fatigue; Consumption of Alcoholic Beverages; Public Health; Young Adult.

\section{Fatiga y consumo de alcohol por estudiantes de pregrado de enfermería}

Objetivo: analizar el uso de alcohol en el patrón de consumo excesivo de alcohol y los niveles de fatiga en estudiantes universitarios de enfermería de la Universidad Federal de Uberlândia. Método: la investigación tiene un enfoque cuantitativo, utilizando estadísticas descriptivas y bivariadas, con un valor de p> 0.05, y realizada de acuerdo con la Resolución 466/12 del CNS, por lo tanto, se aplicaron cuestionarios a una muestra de 202 estudiantes universitarios en enfermería en la Universidad Federal de Uberlândia. Resultados: el estudio mostró un mayor predominio del alcohol en mujeres, con una edad promedio de 22.6 años. Conclusión: el análisis y discusión de los datos permitió afirmar que el abuso de alcohol está presente, principalmente en la forma de binge drinking, que se asocia con la presencia de fatiga y rendimiento académico en estudiantes de licenciatura en enfermería, en diferentes periodos académicos.

Descriptores: Estudiantes de Enfermería; Borrachera; Fatiga; Consumo de Bebidas alcohólicas; Salud Pública; Adulto Joven. 


\section{Introdução}

A iniciação em um curso de graduação na vida dos jovens é um momento marcante, e ocorre em uma fase de significativas mudanças, em um período de desenvolvimento psicossocial, marcado por muitas explorações do novo, de muitas possibilidades, porém, cercado de instabilidades, tornando esse processo de amadurecimento muito complexo(1).

A teoria de desenvolvimento psicossocial caracteriza o jovem em transição da fase de adolescente, já com menor dependência, porém, com pouca autonomia e sem condições de assumir responsabilidades esperadas de um adulto. Acontece uma confusão de identidade em que os principais fatores são a perda de laços familiares e a falta de apoio na tomada de decisões, expectativas da família e do grupo social sobre suas escolhas, dificuldade em lidar com as mudanças, de relacionar com outro grupo social e os processos emocionais na transição do jovem para a fase adulto-jovem ${ }^{(2)}$.

O ambiente universitário é favorável às novas experiências onde os jovens participam de várias comemorações, como a recepção de calouros, festas onde as bebidas são liberadas, eventos com o intuito de arrecadar verba para as formaturas e onde há bares próximos ao campus universitário e à própria residência. Ocorre a oportunidade de conhecer e experimentar as drogas lícitas e ilícitas e propiciar comportamentos de risco, como o padrão de consumo e a frequência de uso de substâncias psicoativas, dirigir embriagado, relações sexuais sem preservativos, violência, problemas no desempenho acadêmico e outros ${ }^{(3)}$.

O consumo de álcool pelos jovens prejudica fisicamente a formação do cérebro, acarreta várias consequências negativas, como a gravidez precoce, e comportamentos eufóricos que causam acidentes e agressões. Quando se inicia o uso de bebidas antes dos 15 anos de idade, aumenta-se em cinco vezes a probabilidade de desenvolver a dependência do álcool e em sete vezes o risco de envolver-se em acidente de trânsito ou em brigas $^{(4)}$.

O álcool é uma droga lícita, composta por substâncias naturais e sintéticas, que alteram a capacidade de agir e raciocinar do indivíduo, cuja produção, distribuição e consumo são aprovados por lei. Sabe-se que o álcool é consumido praticamente em todo o mundo e estima-se uma ingestão média de 6,2 litros de álcool puro por indivíduos de 15 anos ou mais em 2010. O consumo, no Brasil, está em torno de 8,7 litros por pessoa, valor superior à média mundial(5).

No Brasil, o álcool é responsável pelo índice de $63 \%$ da população com cirrose hepática e por $18 \%$ dos acidentes de trânsito em 2012. Acredita que 5,6\% (3\% mulheres e $8 \%$ homens) dos brasileiros correspondem a usuários de abuso e dependência do álcool. A sociedade sofre consequências de forma direta e indireta, seja pelos custos com o sistema de saúde, com os sistemas judiciário, previdenciário, absenteísmo, desemprego, seja pelas mortes associadas ao uso do álcool, em que a faixa etária dos bebedores se encontra entre 20 a 49 anos, por pessoas economicamente ativas ${ }^{(6)}$.

No "I Levantamento Nacional sobre o Uso de Álcool, Tabaco e Outras Drogas" realizado com universitários das capitais brasileiras, ficou demonstrado que $86 \%$ dos alunos fizeram uso de álcool, ao menos, uma vez na vida e $72 \%$ consumiram bebidas no último ano, confirmando o uso recorrente do álcool entre essa população. Um outro dado importante é que a maioria dos entrevistados é composta por acadêmicos da área de humanas ${ }^{(7)}$.

O "binge drinking" ou "beber pesado episódico" é definido, pela Organização Mundial da Saúde (OMS), como a ingestão de $60 \mathrm{~g}$ ou mais de álcool (cerca de 5-6 doses), em uma única ocasião, nos últimos 30 dias. É um padrão de consumo que expõe o indivíduo a um maior risco para danos sociais e de saúde, como prejuízos nas atividades acadêmicas e laborais ${ }^{(8)}$, sendo que esse padrão de abuso de álcool é especialmente comum entre os jovens.

Em pesquisa realizada com estudantes universitários para compreender o que levam os jovens a consumirem bebidas alcoólicas, os motivos mais citados foram: diversão ou prazer; mudança de rotina; sentir os efeitos da droga; reduzir ansiedade; o cansaço e o estresse ${ }^{(6)}$. Nesse sentido, parece que há uma importante correlação entre o uso abusivo de bebidas alcoólicas e a sensação de fadiga por esses graduandos que necessita ser melhor investigada, bem como seus fatores intervenientes.

O estresse diário vivenciado pelos universitários, nas diversas atividades e compromissos pelos quais eles que precisam passar durante o curso, proporciona poucas horas de sono, pois muitos cursam em horário integral e precisam continuar a estudar durante a noite. Outros fatores são: as atividades extracurriculares; o sedentarismo; muitos dizem não ter tempo para realizar atividades físicas; a má alimentação por morarem sozinhos ou em pensionatos e também pela despesa financeira, gerando a fadiga e a sensação de cansaço incessante ${ }^{(9)}$.

A fadiga pode ser avaliada, por vários autores, como um desgaste físico e mental grave e crônico, o que é diferente de cansaço e da falta de motivação devido à atividade física ou a uma enfermidade já diagnosticada. Esse diagnóstico é de difícil conceituação, análise e aferição, além de ser muito preocupante, pois acaba nomeando um estado global oriundo de um resultado desiquilibrado internamente dos sistemas entre a relação do organismo, em que uma alteração de um sistema afeta os demais ${ }^{(10)}$.

Os profissionais de Enfermagem constituem quase metade de toda a força de trabalho da área de saúde no Brasil e no mundo(6-9) e os desgastes vivenciados 
durante a sua formação na graduação, bem como a adoção de hábitos de vida pouco saudáveis, como beber abusivamente, podem refletir negativamente na saúde do futuro trabalhador em médio e longo prazos.

Portanto, o objetivo deste estufo foi analisar o uso de álcool no padrão binge e os níveis de fadiga em graduandos de um curso em Enfermagem de uma universidade pública do Estado de Minas Gerais.

\section{Método}

Trata-se de uma pesquisa descritiva, transversal, com abordagem quantitativa, para identificar a correlação entre o uso de álcool no padrão binge e a fadiga entre os graduandos que cursam Enfermagem em uma universidade pública do Estado de Minas Gerais.

O estudo foi realizado com 440 universitários da Enfermagem nos diferentes períodos do curso. Os critérios de inclusão foram estar regularmente matriculado no curso de graduação em Enfermagem, em qualquer período, e aceitar participar do estudo. Os critérios de exclusão foram estudantes estarem afastados formalmente de suas atividades escolares. Todos os graduandos foram convidados para participar do estudo, no entanto, 202 sujeitos aceitaram participar, perfazendo, portanto, a população deste estudo.

Ao considerar os aspectos éticos referentes à pesquisa envolvendo seres humanos (Resolução CNS no 466/12), este estudo recebeu a autorização do serviço para a sua realização, sendo submetido à avaliação do Comitê de Ética e Pesquisa, com Parecer Consubstanciado final aprovado no 1.315.972, obtido em 10/11/2015. Todos os participantes do estudo foram devidamente esclarecidos sobre a pesquisa, sendo seus direitos e os cuidados a eles garantidos. Após concordarem em participar, assinaram o Termo de Consentimento Livre e Esclarecido (TCLE) em duas vias assinadas pelo pesquisador e participante, sendo uma via do pesquisador e a outra via do participante, conforme regulamentam os dispositivos da Resolução no 466/12 do Conselho Nacional de Saúde (CNS).

Foi realizada a coleta de dados em fevereiro e março de 2017. Para isso, foram realizadas visitas em salas de aula, de diferentes períodos letivos, nas quais se marcaram o dia e o horário para recolher o material de estudo.

O instrumento para a coleta de dados foi constituído por um questionário estruturado, autoaplicável, que continha um roteiro de informações sociodemográficas e os instrumentos Alcohol Use Disorders Identification Test (AUDIT-C) e Dutch Fatigue Scale (DUFS), assim detalhados:

a) Informações sociodemográficas - o questionário tinha a finalidade de caracterizar a amostra do estudo, no qual foram coletadas as variáveis sociodemográficas, incluindo informações referentes ao estado civil, religião, raça, situação ocupacional e educacional com a intenção de evidenciar a caracterização dos graduandos;

b) O AUDIT foi desenvolvido pela OMS no final dos anos oitenta, sendo traduzido e validado para o português do Brasil em 1997. O AUDIT é constituído por dez itens, pontuados em uma escala Likert de zero a quatro, com escores totais de zero a quarenta. Originalmente, era recomendado o ponto de corte oito para avaliar a dependência de álcool. Contudo, estudos posteriores indicaram a necessidade de utilizar pontos de cortes diferentes, pois apresentavam melhores valores de sensibilidade e especificidade(11-12).

Ao considerar a necessidade de instrumentos com aplicação mais rápida, foram desenvolvidas versões abreviadas do AUDIT. As versões breves permitem uma triagem rápida dos transtornos relacionados ao uso de álcool, desse modo, o AUDIT-C é uma das versões mais utilizadas no mundo, correspondendo às três primeiras perguntas do AUDIT ${ }^{(13-14)}$. Sua pontuação total varia de zero a 12 , sendo o ponto de corte cinco, o mais recomendado pela literatura; c) A fadiga foi avaliada pela escala de Dutch Fatigue Scale, desenvolvida por Tiesinga, Dassen e Halfens e publicada em 1998. A escala DUFS mede a fadiga definida como "uma sensação opressiva e sustentada de exaustão e de capacidade diminuída para realizar trabalho físico e mental no nível habitual". Composta por oito itens de avaliação, com respostas tipo Likert de cinco pontos ( 1 a 5), os escores, nos itens, podem ser somados em um escore total que varia de oito a 40 (quanto mais alto o escore, maior a intensidade da fadiga) e a fadiga substancial considerada pela pontuação $>=$ a 14,5 . A fonte de itens para a DUFS foram as características definidoras de fadiga proposta pela North American Nursing Diagnosis Association

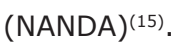

Para a análise estatística, foi elaborado um banco de dados no programa Statistical Program of Social Science (SPSS.20) - version 20 for Windows. Os dados foram apresentados em frequências absoluta ( $\mathrm{n}$ ) e relativa (\%). Os testes estatísticos empregados foram o qui-quadrado (x2), para avaliar a associação das variáveis categóricas, e o coeficiente de correlação de Spearman (Ro), para avaliar a correlação entre duas amostras dependentes. O nível de significância (valor de p) foi estabelecido em 0,05 para todas as análises.

\section{Resultados}

Nessa etapa de estudo, buscaram-se evidenciar, com tabelas, os resultados levantados por meio da estatística simples e aplicada, da população formada por 202 graduandos do curso de Enfermagem, constituído pela maioria do sexo feminino $(n=165)(81,6 \%)$, sendo 
a maioria solteira $(n=151)(91,6 \%)$, de religião católica $(n=78)(47 \%)$, com idade média de 22,6 anos.

A Tabela 1 demonstra que o consumo de álcool em padrão binge se faz presente em 33,7\% $(\mathrm{N}=68)$ dos entrevistados e $37,6 \%(N=76)$ afirmam não fazer esse tipo de uso pesado de álcool. Quase um terço $(28,7 \%)$ dos graduandos não respondeu.

Em relação à frequência do consumo de bebidas, foi observado que $31,7 \%(n=64)$ dos graduandos fazem uso de duas a quatro vezes por mês. Em número de doses por evento, o consumo varia de uma a duas doses contendo álcool, representando 30,7\% $(n=62)$ entre os respondentes. Quando abordados para informar se fazem uso de mais de seis doses em um mesmo evento, 40,6\% dos jovens $(n=82)$ afirmaram nunca terem feito uso dessa quantidade e $29,2 \%(n=59)$ fazem esse tipo de uso em período menor que um mês.

Tabela 1 - Consumo de bebidas alcoólicas e uso em binge drinking pelos graduandos de Enfermagem da UFU* ( $N=202)$. Uberlândia, MG, Brasil, 2017

\begin{tabular}{lc}
\hline Uso de álcool & $\begin{array}{c}\text { Resultados } \\
\mathbf{n}(\%)\end{array}$ \\
\hline Álcool (binge drinking) & $76(37,6)$ \\
Não & $68(33,7)$ \\
Sim & $58(28,7)$ \\
Não respondeu & \\
\hline Frequência de consumo & $53(26,2)$ \\
Nenhum & $63(31,2)$ \\
Uma vez ou menos de uma vez por mês & $64(31,7)$ \\
2 a 4 vezes por mês & $21(10,4)$ \\
2 ou 3 vezes por semana & $0(0)$ \\
4 ou mais vezes por semana & $1(0,5)$ \\
Não respondeu & \\
\hline Número de doses por dia/evento & $62(30,7)$ \\
1 a 2 & $36(17,8)$ \\
\hline 3 a 4 & $($ continua...)
\end{tabular}

\begin{tabular}{lc}
\hline Uso de álcool & $\begin{array}{c}\text { Resultados } \\
\mathbf{n}(\%)\end{array}$ \\
\hline 5 a 6 & $38(18,8)$ \\
7 a 9 & $18(8,9)$ \\
10 ou mais & $22(10,9)$ \\
Não respondeu & $26(12,9)$ \\
\hline Frequência de consumo de mais de seis doses por & \\
dia/evento & \\
Nunca & $82(40,6)$ \\
Menos que mensalmente & $59(29,2)$ \\
Mensalmente & $31(15,3)$ \\
Semanalmente & $19(9,4)$ \\
Diariamente ou quase diariamente & $1(0,5)$ \\
Não respondeu & $10(5,0)$ \\
\hline
\end{tabular}

${ }^{*}$ UFU $=$ Universidade Federal de Uberlândia

A Tabela 2 é representada pelos oitos itens de perguntas da DUFS, que foi utilizada nessa população de 202 universitários e demonstra uma maior média $(3,64)$ na variável DUFS 5 , que se refere à "necessidade de descansar mais". A segunda maior média foi encontrada na variável DUFS 2 referente a: "precisam de mais energia para dar conta das tarefas diárias", no valor de 3,20.

No final da mesma tabela, é representado o escore total da escala DUFS, que demonstra uma variação de oito a 40, em que o valor mínimo obtido corresponde à menor pontuação possível e, assim, ao menor índice de fadiga. A pontuação máxima de 39,00 aproxima-se do limite da pontuação, indicando que, quanto maior o escore, mais intensos os níveis da fadiga. Neste estudo, a média de fadiga encontrada foi de 22,48 , o que indica que a fadiga está presente e com intensidade significativa, podendo causar prejuízos aos graduandos. Viu-se, considerando essa média, que 70,3\% $(n=142)$ dos universitários apresentam fadiga. O desvio-padrão alto mostra variação nos níveis de fadiga dos entrevistados.

Tabela 2 - Itens da escala de fadiga da DUFS* entre graduandos de Enfermagem da Universidade Federal de Uberlândia (N=202). Uberlândia, MG, Brasil, 2017

\begin{tabular}{|c|c|c|c|c|}
\hline Item & $\begin{array}{c}\mathrm{N}^{\circ} \\
(202)\end{array}$ & Mínimo - Máximo & Média & Desvio-Padrão \\
\hline DUFS 1 (sensação forte e constante de falta de energia?) & 199 & $1-5$ & 2,74 & 1,538 \\
\hline DUFS* 2 (mais energia para dar conta das tarefas diárias?) & 199 & $1-5$ & 3,20 & 1,527 \\
\hline DUFS* 3 (se sentido sem disposição para fazer as coisas?) & 200 & $1-5$ & 2,95 & 1,418 \\
\hline DUFS* 4 (acordado com a sensação de exausto e desgastado?) & 198 & $1-5$ & 3,18 & 1,602 \\
\hline DUFS $^{\star} 5$ (necessidade de descansar mais?) & 200 & $1-5$ & 3,64 & 1,491 \\
\hline DUFS* 6 (conseguindo fazer suas atividades do dia a dia?) & 199 & $1-5$ & 2,08 & 1,216 \\
\hline DUFS 7 (sua vontade de ter relação sexual diminuiu?) & 196 & $1-5$ & 1,80 & 1,171 \\
\hline DUFS 8 (difícil de concentrar em uma coisa por muito tempo?) & 198 & $1-5$ & 2,74 & 1,481 \\
\hline Escala DUFS ${ }^{*}$ da fadiga (TOTAL) & & 8-39 & 22,4815 & 8,15384 \\
\hline
\end{tabular}

*DUFS = Dutch Fatigue Scale 
A Tabela 3, ao comparar os itens da DUFS pela variável sexo, demonstra que houve diferenças estatisticamente maiores para o sexo feminino nas seguintes afirmações: "acordar com a sensação de exausto e desgastado" $(p=0,020)$ e "diminuiu a vontade de ter relação sexual" $(p=0,038)$.

Tabela 3 - Associação DUFS* e a variável sexo ( $N=202)$. Uberlândia, MG, Brasil, 2017

\begin{tabular}{|c|c|c|c|c|}
\hline Item & Sexo & n (202) & Média & Valor de $p$ \\
\hline DUFS* 1 (sensação forte e constante de falta de energia?) & $\begin{array}{l}\text { Feminino } \\
\text { Masculino }\end{array}$ & $\begin{array}{c}173 \\
26\end{array}$ & $\begin{array}{l}02,26 \\
84,96\end{array}$ & 0,142 \\
\hline DUFS $^{*} 2$ (mais energia para dar conta das tarefas diárias?) & $\begin{array}{l}\text { Feminino } \\
\text { Masculino }\end{array}$ & $\begin{array}{c}173 \\
26\end{array}$ & $\begin{array}{c}101,25 \\
91,67\end{array}$ & 0,417 \\
\hline DUFS 3 (se sentido sem disposição para fazer as coisas?) & $\begin{array}{l}\text { Feminino } \\
\text { Masculino }\end{array}$ & $\begin{array}{c}174 \\
26\end{array}$ & $\begin{array}{c}102,23 \\
88,92\end{array}$ & 0,264 \\
\hline DUFS 4 (acordado com a sensação de exausto e desgastado?) & $\begin{array}{l}\text { Feminino } \\
\text { Masculino }\end{array}$ & $\begin{array}{c}172 \\
26\end{array}$ & $\begin{array}{c}103,08 \\
75,83\end{array}$ & $0,020^{\dagger}$ \\
\hline DUFS $^{\star} 5$ (necessidade de descansar mais?) & $\begin{array}{l}\text { Feminino } \\
\text { Masculino }\end{array}$ & $\begin{array}{c}174 \\
26\end{array}$ & $\begin{array}{c}101,97 \\
90,69\end{array}$ & 0,332 \\
\hline DUFS $^{*} 6$ (conseguindo fazer suas atividades do dia a dia?) & $\begin{array}{l}\text { Feminino } \\
\text { Masculino }\end{array}$ & $\begin{array}{c}174 \\
25\end{array}$ & $\begin{array}{c}99,42 \\
104,06\end{array}$ & 0,691 \\
\hline DUFS 7 (sua vontade de ter relação sexual diminuiu?) & $\begin{array}{l}\text { Feminino } \\
\text { Masculino }\end{array}$ & $\begin{array}{c}170 \\
26\end{array}$ & $\begin{array}{c}101,41 \\
79,48\end{array}$ & $0,038^{\dagger}$ \\
\hline DUFS $^{*} 8$ (difícil de concentrar em uma coisa por muito tempo?) & $\begin{array}{l}\text { Feminino } \\
\text { Masculino }\end{array}$ & $\begin{array}{c}172 \\
26\end{array}$ & $\begin{array}{c}100,63 \\
92,02\end{array}$ & 0,464 \\
\hline
\end{tabular}

${ }^{*}$ DUFS = Dutch Fatigue Scale; ${ }^{\dagger}$ Associação Significativa no Nível de Significância do Teste Qui-Quadrado

Na Tabela 4, percebe-se uma correlação positiva entre a frequência de consumo de bebidas alcoólicas e o número de reprovações ( $p=0,001)$, entre a frequência em que se consomem seis ou mais doses de bebidas alcoólica em uma ocasião e o número de reprovações $(p=0,006)$ e também no uso de álcool em padrão binge e o número de reprovações $(p=0,022)$. Também se nota uma correlação negativa entre o uso de álcool em padrão binge e o período letivo $(p=0,018)$.

Tabela 4 - Correlação entre as variáveis do AUDIT* C e o número de reprovações, período letivo e idade $(N=202)$. Uberlândia, MG, Brasil, 2017

\begin{tabular}{|c|c|c|c|c|}
\hline Item & & Reprovações & $\begin{array}{c}\text { Semestre } \\
\text { Letivo }\end{array}$ & Idade \\
\hline Qual é a frequência do seu consumo de bebidas alcoólicas? & $\begin{array}{c}\text { Ro }= \\
p=\end{array}$ & $\begin{array}{l}0,323 \\
0,001^{\dagger}\end{array}$ & $\begin{array}{l}-, 089 \\
0,265\end{array}$ & $\begin{array}{l}0,028 \\
0,700\end{array}$ \\
\hline $\begin{array}{l}\text { Quantas doses contendo álcool você consome num dia típico quando } \\
\text { você está bebendo? }\end{array}$ & $\begin{array}{c}\text { Ro }= \\
p=\end{array}$ & $\begin{array}{l}0,165 \\
0,100\end{array}$ & $\begin{array}{l}-, 162 \\
0,053\end{array}$ & $\begin{array}{r}-0,115 \\
0,140\end{array}$ \\
\hline $\begin{array}{l}\text { Qual é frequência que você consome seis ou mais doses de bebidas } \\
\text { alcoólicas em uma ocasião? }\end{array}$ & $\begin{array}{c}\text { Ro }= \\
p=\end{array}$ & $\begin{array}{l}0,269 \\
0,006^{+}\end{array}$ & $\begin{array}{c}-0,149 \\
0,65\end{array}$ & $\begin{array}{r}-0,024 \\
0,749\end{array}$ \\
\hline Binge (valor total) & $\begin{array}{c}\mathrm{Ro}= \\
\mathrm{p}=\end{array}$ & $\begin{array}{c}0,230 \\
0,022^{\dagger}\end{array}$ & $\begin{array}{l}-0,200 \\
0,018^{\dagger}\end{array}$ & $\begin{array}{r}-0,077 \\
0,327\end{array}$ \\
\hline
\end{tabular}

${ }^{*}$ AUDIT $=$ Alcohol Use Disorders Identification Test $;{ }^{\dagger}$ Associação Significativa no Nível de Significância do Teste Qui-Quadrado

Na Tabela 5, observa-se uma correlação negativa entre a idade e o item quatro da escala DUFS, apontando que, quanto mais jovem, maior o sentimento de "ter acordado com a sensação de estar exausto e desgastado" $(p=0,043)$.
Também foram encontradas correlações negativas entre o item oito da escala DUFS e a idade e o período letivo, mostrando que, quanto maior a idade e maior o período letivo, mais tem sido "difícil de concentrar em uma coisa por muito tempo" $(p=0,046)$. 
Tabela 5 - Correlação entre as variáveis do DUFS* e o número de reprovações, período letivo e idade (=202). Uberlândia, MG, Brasil, 2017

\begin{tabular}{|c|c|c|c|c|}
\hline Item & & Reprovações & $\begin{array}{c}\text { Semestre } \\
\text { Letivo }\end{array}$ & Idade \\
\hline \multirow{2}{*}{ DUFS ${ }^{*} 1$} & $\mathrm{Ro}=$ & $-0,082$ & 0,071 & $-0,064$ \\
\hline & $p=$ & 0,397 & 0,378 & 0,385 \\
\hline \multirow{2}{*}{ DUFS* 2} & $\mathrm{Ro}=$ & 0,133 & 0,015 & 0,029 \\
\hline & $p=$ & 0,164 & 0,848 & 0,696 \\
\hline \multirow{2}{*}{ DUFS 3} & $\mathrm{Ro}=$ & $-0,009$ & 0,096 & $-0,029$ \\
\hline & $p=$ & 0,930 & 0,231 & 0,976 \\
\hline \multirow{2}{*}{ DUFS 4} & $\mathrm{Ro}=$ & 0,114 & $-0,079$ & $-0,149$ \\
\hline & $p=$ & 0,164 & 0,328 & $0,043^{\dagger}$ \\
\hline \multirow{2}{*}{ DUFS 5} & $\mathrm{Ro}=$ & $-0,041$ & $-0,082$ & $-0,083$ \\
\hline & $p=$ & 0,668 & 0,307 & 0,260 \\
\hline \multirow{2}{*}{ DUFS* 6} & $\mathrm{Ro}=$ & $-0,125$ & $-0,028$ & $-0,043$ \\
\hline & $p=$ & 0,195 & 0,728 & 0,558 \\
\hline \multirow{2}{*}{ DUFS* 7} & Ro $=$ & $-0,84$ & $-0,059$ & $-0,021$ \\
\hline & $p=$ & 0,384 & 0,465 & 0,775 \\
\hline \multirow{2}{*}{ DUFS 8} & $\mathrm{Ro}=$ & 0,56 & 0,288 & 0,147 \\
\hline & $p=$ & 0,562 & $0,000^{\dagger}$ &, $046^{+}$ \\
\hline \multirow{2}{*}{$\begin{array}{l}\text { Fadiga } \\
\text { (Total) }\end{array}$} & $\mathrm{Ro}=$ & $-0,87$ & ,051 & $-0,049$ \\
\hline & $p=$ & 0,375 & 0,529 & 0,514 \\
\hline
\end{tabular}

${ }^{*}$ DUFS = Dutch Fatigue Scale $;{ }^{\dagger}$ Associação Significativa no Nível de Significância do Teste Qui-Quadrado

\section{Discussão}

As substâncias psicoativas (SPA) ou drogas psicotrópicas são substâncias que agem no Sistema Nervoso Central (SNC), alterando suas funções e, consequentemente, produzindo efeitos psíquicos e comportamentais no indivíduo(16). O álcool pode ser classificado como uma substância psicoativa que exerce função depressora do SNC(17). Dentre as substâncias, o álcool e o tabaco merecem uma atenção especial devido à prevalência de usuários no mundo e os elevados custos sociais, econômicos e à saúde que acarretam(5).

Esta pesquisa denominou o perfil de consumo de SPA de uma população jovem com idade média de 22,6 anos, universitária e com predominância do gênero feminino $(81,6 \%)$, como na pesquisa realizada em um curso de Enfermagem onde as mulheres representavam a maioria $(87,13 \%)$, mantendo as características históricas da profissão de Enfermagem, pois a procura maior para esse curso é de mulheres ${ }^{(18-20)}$.

Os acadêmicos de Enfermagem fazem parte desse contexto do consumo de álcool. Em face disso, deve ser dispensada uma atenção especial aos graduandos, na qual, nesta investigação, 33,7\% admitiram o uso pesado do álcool - binge drinking (mais de seis doses num evento) demonstrado na Tabela 1 . No estudo realizado em graduandos de Enfermagem na cidade de Ribeirão Preto, 35\% relataram já ter consumido mais que seis doses em uma única ocasião, pelo menos, uma vez no último ano, caracterizando o binge drinking ${ }^{(21)}$.
O consumo de álcool pelos estudantes, de acordo com o AUDIT, identificou que a maioria dos estudantes $(82,9 \%)$ é abstinente ou fez uso de baixo risco nos últimos 12 meses $^{(22)}$. Entretanto, 17,1\% dos estudantes enquadraram-se no grupo de risco, demonstrando um consumo considerado prejudicial e com possíveis consequências negativas. Em outro estudo sobre o uso do tipo binge, esse padrão de beber excessivo, observado entre os homens do estudo $(42,6 \%)$, foi superior ao dos estudantes universitários de levantamento nacional cuja prevalência foi de $31,3 \%{ }^{(7)}$. Entretanto, as mulheres apresentaram uma prevalência de binge muito próxima entre esse estudo e os outros realizados(7,18).

Na Tabela 1, observa-se uma prevalência alta de consumo de álcool nos últimos três meses (73,3\%), semelhantemente à encontrada em outra pesquisa, que mostra o uso de álcool nas 107 maiores cidades do Brasil, com índice de $74,6 \%{ }^{(23)}$.Levantamento semelhante identificou uma população de $84 \%$ de estudantes que já haviam feito uso de álcool(24).

Esses dados confirmam a posição do Ministério da Saúde em considerar o uso abusivo e a dependência de álcool e outras drogas uma questão que precisa ter uma abordagem social, psicológica, econômica e política, não se restringindo aos aspectos meramente clínicos $^{(19)}$.

A população formada pelos adultos jovens apresenta maior risco para o consumo do álcool, pois o acadêmico, que experimentou essa substância, tende a continuar o seu uso até recentemente (último mês), como mostram os dados de que $31,2 \%$ dos entrevistados bebem, ao menos, uma vez por mês (Tabela 1). Entre universitários do município de Ribeirão Preto - SP, o percentual foi maior, de $57,5 \%$, no qual se identifica o consumo de álcool uma vez ao mês ${ }^{(21)}$.

Os jovens têm sido o público-alvo das propagandas e dos merchandisings das indústrias de consumo e lazer por serem potenciais consumidores das substâncias psicoativas. Essa condição pode agravar-se com o acesso à universidade, considerando uma nova fase em que a independência e a liberdade estarão fora do círculo familiar, particularmente para aqueles estudantes que se deslocam para outras cidades maiores que tenham o curso universitário e, na busca de integração grupal e de novas experiências, podem desencadear o consumo de drogas lícitas e ilícitas ${ }^{(19)}$.

Na Tabela 2, a maior média na escala DUFS foi de 3,64 na variável que se refere à "necessidade de descansar mais", sendo esses motivos citados abaixo como realidade também vivida pelos graduandos desta pesquisa. A maioria dos cursos de graduação em Enfermagem tem, em seu projeto pedagógico, aulas em período integral, congressos, seminários, estágios, aulas práticas, além de projetos de ensino e pesquisas e outros mais. Por ser difícil realizar todas as atividades planejadas, acaba 
não restando muito tempo para outros compromissos, como o lazer, compromissos religiosos, atividade física, dormir e repousar, principalmente, para os estudantes que trabalham ou realizam estágios extracurriculares ${ }^{(1)}$.

$\mathrm{Na}$ mesma tabela, observou-se que $70,3 \%$ dos universitários sentem fadiga. Esse dado foi observado em outra pesquisa na qual $83,5 \%$ dos seus estudantes da graduação de Enfermagem apresentaram também queixas de fadiga(9).

Em estudo similar, a maioria dos estudantes era do sexo feminino e com idade média de 21,6 anos e com ocupações extracurriculares; elas estavam com sobrepeso e cerca de $20 \%$ com sinais de depressão e disforia nos quais a fadiga foi menos intensa no primeiro ano letivo ${ }^{(9)}$. Eram estudantes de 18 a 23 anos de idade, em sua maioria, a partir do terceiro período letivo da graduação de Enfermagem, momento em que os alunos iniciaram suas aulas práticas supervisionadas e as mudanças de ambientes e rotinas causaram um maior cansaço físico e mental, comprometendo os seus desempenhos acadêmicos $^{(1)}$.

Já no final da Tabela 2, neste estudo, é apresentada uma média de fadiga dentre os graduandos com valor de 22,15 no qual se verifica que a fadiga está presente em grande parte dos entrevistados e com variados níveis, o que pode estar relacionado ao período letivo e à idade.

A fadiga é um estado que pode estar presente em pessoas saudáveis ou não, sendo um fenômeno multifatorial, multidimensional e subjetivo. Ela é considerada um cansaço que não melhora com o repouso ou outro meio de revigorar as energias. Causa limitações no processo de aprendizagem dos estudantes universitários, prejudicando a formação profissional e a qualidade de vida(9).

Em pesquisa, com o tema da qualidade de vida dos estudantes de graduação, com relatos e acompanhamento de fatores encontrados no cotidiano do processo de ensino-aprendizagem que repercutem diretamente na saúde desses universitários que iniciam a vida acadêmica, demonstraram-se forte pressão e estresse desde o processo para ingressar na faculdade ${ }^{(1)}$.

A Tabela 3 mostra a variável sexo em que se percebe, na população feminina, a DUFS $4(p=0,020)$ referente a "acordar com a sensação de exausto e desgastado" e também a DUFS $7(p=0,038)$ em que as mulheres não "diminuíram a vontade de ter relação sexual" por estarem fadigadas. As estudantes do sexo feminino referenciam as maiores médias de alterações na qualidade de vida, como também de alterações nos aspectos emocional e mental, pela rotina vivenciada na graduação de Enfermagem ${ }^{(1)}$.

Resultado preocupante, encontrado neste estudo e representado na Tabela 5, mostra que, quanto mais reprovações, maior a frequência de consumo de álcool e, principalmente, o consumo em binge. $\mathrm{O}$ uso abusivo do álcool está diretamente relacionado às dificuldades de como agir diante das mudanças e na adaptação dos universitários em lidar com problemas como reprovação, rotinas e relacionamentos conturbados ${ }^{(24)}$.

Situação semelhante foi encontrada em outra pesquisa na qual se demonstrou que $33,6 \%$ dos alunos pesquisados tiveram reprovações em disciplinas, podendo isso ser explicado pelo fato de que, quando ocorre o abuso do álcool, os alunos tendem a dormir em sala de aula, faltam às aulas e, consequentemente, sofrem reprovações pelo mau desempenho acadêmico(20).

Estudo realizado com 189 acadêmicas do curso de Enfermagem identificou que, quanto menor a idade, mais intensos foram os sintomas de fadiga(9). Segundo o estudo, mesmo os índices de fadiga sendo elevados entre os universitários, não causaram grandes prejuízos aos entrevistados, pois eles conseguiram trabalhar de formar individual para reduzir a fadiga e diminuir os danos aos seus compromissos e ao desempenho nas atividades acadêmicas e extracurriculares ${ }^{(9)}$. Importante pontuar que essa correlação também foi demonstrada nesta pesquisa, na Tabela 5, em que, quanto menor a idade, mais significativa essa sensação de "ter acordado com a sensação de estar exausto e desgastado".

\section{Conclusão}

Os resultados deste estudo trazem dados preocupantes quanto ao uso de álcool em binge por estudantes de curso de Enfermagem. A pesquisa também mostrou que o uso de álcool não está relacionado à fadiga, mas encontra correlação com seu desempenho acadêmico. Nesse mesmo sentido, o sentimento de fadiga parece estar correlacionado com a idade do estudante.

Os estudantes da graduação vivenciam, no processo de ensino e aprendizagem, uma sobrecarga das atividades curriculares, principalmente quando o curso for integral, conjuntamente com forte pressão e estresses nas variadas relações sociais. É preciso que o estudante saiba lidar com as situações que estão causando a fadiga e agir de maneira a reduzir o estresse, conseguir sentir-se descansado e disposto para as atividades diárias.

Percebe-se que é preciso repensar na maneira de atuar na atenção preventiva do uso do álcool e outras drogas e nas situações estressoras que causam fadiga, antes e durante a graduação. Ressalta-se, tendo em vista a relevância do tema, que os resultados deste estudo podem fornecer subsídios para ações e programas de promoção, prevenção e tratamento adequados às diferentes necessidades da população em questão.

\section{Referências}

1. Osse MC, Costa II da. Mental health and quality of life at a student hall of residence at the Universidade de Brasília, 
Brazil. Estud Psicol. 2011;28(1):115-22. doi: http://doi. org/10.1590/S0103-166X2011000100012

2. Erikson H. Identity, youth and crisis. New York: WW Norton Company; 1968

3. Kerr-Corrêa F, Andrade AG de, Bassit AZ, Boccuto NMVF. Alcohol and drug use by Unesp medical students. Rev Bras Psiquiatr. 1999;21(2):95-100. doi: http://doi. org/10.1590/S1516-44461999000200005

4. World Health Organization. Global strategy to reduce the harmful use of alcohol. $1^{\text {st }}$ ed. Geneva: World Health Organization; 2010 [cited Nov 28, 2017]. Available from: http://apps.who.int/iris/ bitstream/10665/44395/1/9789241599931_eng. pdf?ua $=1 \& u a=1$

5. World Health Organization. Global status report on alcohol and health 2014. Luxembourg: World Health Organization; 2014 [cited Nov 28, 2017]. Available from: http://apps. who.int/iris/bitstream/10665/112736/1/9789240692763_ eng.pdf

6. Baumgarten LZ, Gomes VLO, Fonseca AD da. Alcohol consumption among university students in the health area of Federal University of Rio Grande/RS: subsidy to the nursing. Esc Anna Nery. 2012;16(3):530-5. [cited Nov 28, 2017]. Available from: http://www.scielo.br/scielo. php?script=sci_arttext\&pid $=$ S1414-81452012000300015 7. Ministério da Saúde. Secretaria Nacional de Políticas sobre Drogas (BR). I Levantamento Nacional sobre o Uso de Álcool, Tabaco e Outras Drogas entre Universitários das 27 Capitais Brasileiras/Secretaria Nacional de Políticas sobre Drogas. Brasília: Presidência da República, Secretaria Nacional de Políticas sobre Drogas; 2010 [Acesso 28 nov 2017]. Disponível em: http://www.grea. org.br/userfiles/GREA-ILevantamentoNacionalUniversit arios.pdf

8. Pinsky I, Zaleski M, Laranjeira R, Caetano R. Primeiro Levantamento Nacional sobre os Padrões de Consumo de Álcool na População Brasileira. Rev Bras Psiquiatr. 2010;32(3):214-5. doi: http://doi.org/10.1590/ S1516-44462010000300003

9. Amaducci CM, Mota DDC, Pimenta CAM. Fatigue among nursing undergraduate students. Rev Esc Enferm USP. 2010;44(4):1052-8. doi: http://doi.org/10.1590/ S0080-62342010000400028

10. Oliveira JRS, Viganó M, Lunardelli MCF, Canêo LC, Goulart E Júnior. Fadiga no trabalho: como o psicólogo pode atuar? Psicol Estud. 2010;15(3):633-8. doi: http:// doi.org/10.1590/S1413-73722010000300021

11. Reinert DF, John A. The alcohol use disorders identification test: an update of research findings. Alcohol Clin Exp Res. 2007;31(2):185-99. doi: http:// doi.org/10.1111/j.1530-0277.2006.00295.x/full

12. Meneses-Gaya C de, Zuardi AW, Loureiro SR, Crippa JAS. Alcohol Use Disorders Identification Test (AUDIT): an updated systematic review of psychometric properties.
Psychol Neurosci. 2009; 2(1):83-97. doi: http://doi. org/10.3922/j.psns.2009.1.12

13. Aertgeerts B, Buntinx F, Ansoms S, Fevery J. Questionnaires are better than laboratory tests to screen for current alcohol abuse or dependence in a male inpatient population. Acta Clin Belg. 2002;57(5):241-9. doi: http://doi.org/10.1179/acb.2002.048

14. Bischof G, Grothues J, Reinhardt S, John U, Meyer C, Ulbricht S, et al. Alcohol Screening in General Practices Using the AUDIT: How Many Response Categories Are Necessary? Eur Addic Res. 2007;13(1):25-30. doi: http:// doi.org/10.1159/000095812

15. Tiesinga LJ, Dassen TWN, Halfens RJG. DUFS and DEFS: development, reliability and validity of the Dutch Fatigue Scale and the Dutch Exertion Fatigue Scale. Int J Nurs Stud. 1998;35(1-2):115-23. doi: http://doi. org/10.1016/S0020-7489(98)00005-4

16. Dalgalarrondo P. Psicopatologia e Semiologia dos Transtornos Mentais. 2. ed. São Paulo: Artmed; 2009

17. Nery A Filho, Torres IMAP. Drugs: does it matter?: check here. Salvador: Universidade Federal da Bahia; 2002

18. Fachini A, Furtado EF. Alcohol use and drinking expectations among college students: an analysis of sex differences. Psicol Teor Pesqui. 2013;29(4):421-8. doi: http://doi.org/10.1590/S0102-37722013000400008

19. Picolotto E, Libardoni LFC, Migott AMB, Geib LTC. Prevalence and factors associated with psychoactives substances consumption for academics of nursing of the University of Passo Fundo. Ciênc Saúde Coletiva. 2010;15(3):645-54. doi: http://doi.org/10.1590/ S1413-81232010000300006

20. Junqueira MAB, Antunes AV, Oliveira VC de. Standard of alcohol use and academic performance among students of nursing course of Federal University of Uberlândia, MG, Brasil. Biosci J. 2016;32(6):1649-56. doi: http:// doi.org/10.14393/BJ-v32n1a2016-28395

21. Pillon S, Webster C. Teste de identificação de transtornos relacionados ao uso de álcool (AUDIT) entre estudantes universitários. Rev Enferm UERJ. [Internet]. 2006;14(3):325-32. [Acesso 30 nov 2017]. Disponível em: http://www.facenf.uerj.br/v14n3/v14n3a01.pdf

22. Silva ÉC, Tucci AM. Cross-sectional study on the risk of alcohol use in a sample of students in a Brazilian federal university. J Bras Psiquiatr. 2014;63(4):317-25. doi: http://doi.org/10.1590/0047-2085000000040

23. Pedrosa AAS, Camacho LAB, Passos SRL, Oliveira RVC de. Alcohol consumption by university students. Cad Saúde Pública. 2011;27(8):317-25. doi: http://doi. org/10.1590/S0102-311X2011000800016

24. Kerr-Corrêa F, Simão MO, Martins RA. Prevenção ao uso de álcool por estudantes universitários [Internet]. 2003. [Acesso 30 nov 2017]. Disponível em: http://fmb. unesp.br/Home/Departamentos/Neurologia,PsicologiaeP 
siquiatria/ViverBem/Prevencao_ao_uso_de_alcool_por_ estudantes_universitarios.pdf

\section{Contribuição dos autores}

Concepção e desenho da pesquisa: Marcelle Aparecida de Barros Junqueira. Obtenção de dados: Nathálie Dias de Oliveira Silva, Gabriel Fernandes Machado. Análise e interpretação dos dados: Nathálie Dias de Oliveira Silva, Gabriel Fernandes Machado. Análise estatística: Gabriel Fernandes Machado. Obtenção de financiamento: Marcelle Aparecida de Barros Junqueira. Redação do manuscrito: Nathálie Dias de Oliveira Silva. Revisão crítica do manuscrito quanto ao conteúdo intelectual importante: Marcelle Aparecida de Barros Junqueira.

Todos os autores aprovaram a versão final do texto.

Conflito de interesse: os autores declararam que não há conflito de interesse.

Esta licença permite que outros distribuam, remixem, adaptem e criem a partir do seu trabalho, mesmo para fins comerciais, desde que the atribuam o devido crédito pela criação original. É a licença mais flexível de todas as licenças disponíveis. É recomendada para maximizar a disseminação e uso dos materiais licenciados. 